International Journal of Pure and Applied Mathematics

Volume 93 No. 3 2014, 389-397

ISSN: 1311-8080 (printed version); ISSN: 1314-3395 (on-line version)

url: http://www.ijpam.eu

doi: http://dx.doi.org/10.12732/ijpam.v93i3.9

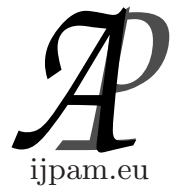

\title{
ON SUBSECTION METHOD OF A PSC MEDIUM OF EM ON A TWO LAYERED GROUND MODEL
}

\author{
Panyawat Haarsa ${ }^{1}$, Supaporn Pothat ${ }^{2}$ \\ ${ }^{1}$ Department of Mathematics \\ Srinakharinwirot University \\ Bangkok, 10110, THAILAND \\ ${ }^{2}$ Wad Ban-Koh School \\ Bandara, Amphoe Pichai \\ Uttaradit, 53220, THAILAND
}

\begin{abstract}
The goal of this paper is to investigate the structure of the earth by dividing it into two layers with a positively skewed curve in overburden and a constant in host medium. The subsection method of the ground is used mathematically to find the electric fields in overburden. Numerical solutions are computed to show graphically the behaviors of the electric field. The responses of electric field are relevant by a positively skewed curve medium ground profile.
\end{abstract}

\section{AMS Subject Classification: 86A25}

Key Words: electromagnetic field, subsection, positively skewed curve, Chave's algorithm, two layered ground, Hankel transform

\section{Introduction}

The electromagnetic prospecting technique is the most generally used in mineral exploration because it responses best to a good electrical conductors at a shallow depth to deep as many kilometers from the ground surface, and it is far less costly than other investigation methods. Lee and Ignetik [3] considered the forward problem of the transient electromagnetic response of a half-space with

Received: December 28, 2013

(c) 2014 Academic Publications, Ltd.

$\S_{\text {Correspondence author }}$ url: www.acadpubl.eu 
an exponentially varying conductivity profiles. They pointed out that the conductivity variation of the ground may sometimes be reasonably approximated by an exponential variation since soil salinity profiles frequently show monotonically increasing or decreasing salt conductivity of the ground. Yooyuanyong [5] gave the mathematical model of electromagnetic sounding for a conductive thin disc embedded in an inhomogeneous ground.

In this paper, we present a mathematical model and techniques for studying the structure of the earth's surface layers. We consider the ground containing two layers, the overburden and host medium. The conductivity of overburden given by $\sigma_{o v}(z)=\left(\sigma_{0}+z\right) e^{-b z / 2}, 0 \leq z \leq d$, where $\sigma_{0}$ is a positive constant, $b$ is constant, $d$ is the thickness of overburden. The conductivity of host medium, $z \geq d$, is a constant given by $\sigma_{h o}(z)=\sigma_{0}$. The conductivity ground profile in this paper is different from the model used by Lee and Ignetik [3] and Yooyuanyong [5].

\section{Formulation and Solution of the Problem}

A cylindrical polar coordinates $(r, \theta, z)$ is introduced with $z>0$ which is taken vertically positive downwards, and the origin under the center of the horizontal circular loop is used. The azimuthally symmetry gives merely electric field $E_{\theta}$, and magnetic fields $H_{r}$, and $H_{z}$ components. Following Morrison et al., [4], these field quantities are found to satisfy Maxwell's equations (Hohmann and Raiche, [2]) in the form of equations

$$
\begin{gathered}
i \omega \mu H_{r}=-\frac{\partial E}{\partial z}, \\
i \omega \mu H_{z}=\frac{1}{r} \frac{\partial(r E)}{\partial r},
\end{gathered}
$$

and

$$
\frac{\partial H_{r}}{\partial z}-\frac{\partial H_{z}}{\partial r}=(\sigma(z)-i \omega \varepsilon) E+J_{s}
$$

where $i=\sqrt{-1}$ is an imaginary number, $J_{s}=a I(\omega) \delta(r-a) \delta(z+h) / r$ is the source current density, $\omega$ is the angular frequency, $\delta$ is the delta function, $\sigma(z)$ is the electrical conductivity of medium depending on depth only, $\varepsilon$ is the electric permittivity of medium, $\mu$ is the magnetic permeability of medium, and $I(\omega)$ is the current in a coil of a small radius $a$. Eliminating $H_{r}$ and $H_{z}$ from above equations lead to get the differential equation for electric fields as.

$$
i \omega \mu J_{s}=-\frac{\partial^{2} E}{\partial z^{2}}-\frac{\partial^{2} E}{\partial r^{2}}-\frac{1}{r} \frac{\partial E}{\partial r}+\frac{E}{r^{2}}-\left(i \omega \mu \sigma(z)+\omega^{2} \mu \varepsilon\right) E .
$$


Taking Hankel transform which is defined as

$$
\widetilde{E}(\lambda, z, \omega)=\int_{0}^{\infty} r J_{1}(\lambda r) E(r, z, \omega) d r,
$$

where $J_{1}$ is Bessel function of the first kind of order 1, and equation (4) becomes

$$
i \omega \mu a I(\omega) \delta(z+h) J_{1}(\lambda a)=-\frac{\partial^{2} \widetilde{E}}{\partial z^{2}}+\left(\lambda^{2}-\left(i \omega \mu \sigma(z)+\omega^{2} \mu \varepsilon\right)\right) \widetilde{E} .
$$

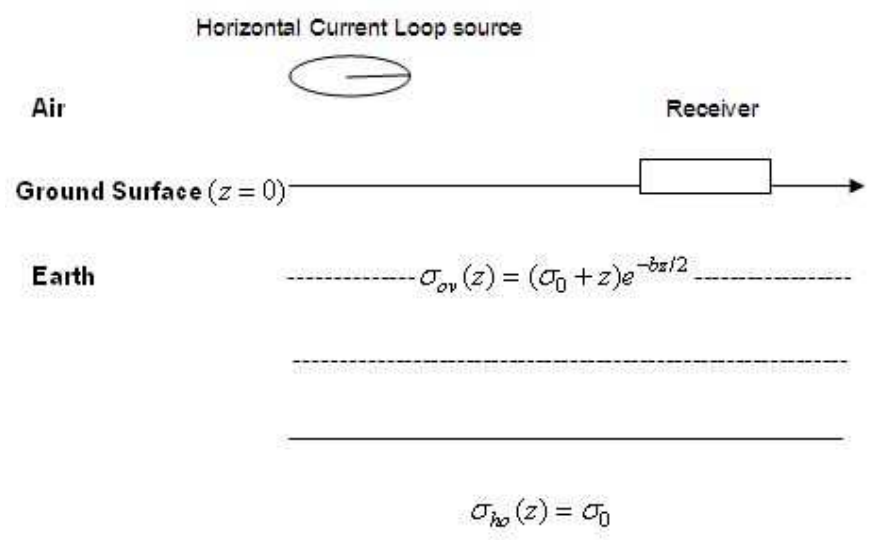

Figure 1: Illustration of a two layered ground model with a positively skewed curve medium in overburden

The electric field in air can be denoted by $\widetilde{E}_{a i}(\lambda, z, \omega)$ and expressed as the sum of two components,

$$
\widetilde{E}_{a i}(\lambda, z, \omega)=\widetilde{E}^{p}(\lambda, z, \omega)+\widetilde{E}^{s}(\lambda, z, \omega),
$$

where $\widetilde{E}^{p}(\lambda, z, \omega)$ is the primary field and $\widetilde{E}^{s}(\lambda, z, \omega)$ is the secondary field. Both electric fields can be obtained from equation (5). In air, $\sigma_{a i}(z) \cong 0$ and the electric field is given by

$$
\widetilde{E}_{a i}(\lambda, z, \omega)=\frac{i \omega \mu_{0} a I(\omega) J_{1}(\lambda a) e^{-\lambda|z+h|}}{2 \lambda}+\kappa e^{\lambda z}, z \leq 0
$$

which remains bounded as $z \rightarrow-\infty$, and $\kappa$ is arbitrary constant to be determined. 
From equation (4), we can obtain the partial differential equation for the electric filed in overburden as

$$
\begin{aligned}
& \frac{\partial^{2} E_{o v}(r, z, \omega)}{\partial z^{2}}+\frac{\partial^{2} E_{o v}(r, z, \omega)}{\partial r^{2}} \\
& +\frac{1}{r} \frac{\partial E_{o v}(r, z, \omega)}{\partial r}-\frac{E_{o v}(r, z, \omega)}{r^{2}}+k_{o v}^{2} E_{o v}(r, z, \omega)=0 .
\end{aligned}
$$

where $E_{o v}(r, z, \omega)$ is the electric field in ground, $k_{o v}^{2}=i \omega \mu_{o} \sigma(z)+\omega^{2} \mu_{o} \varepsilon_{o}$, $\mu_{o}$ is the magnetic permeability of ground, $\varepsilon_{O}$ is the electric permittivity of ground and $\sigma(z)=\left(\sigma_{0}+z\right) e^{-b z / 2}$ is the conductivity of ground. Taking Hankel transform to equation (7), we obtain

$$
\frac{d^{2} \widetilde{E}_{o v}(\lambda, z, \omega)}{d z^{2}}-m_{o v}^{2} \widetilde{E}_{o v}(\lambda, z, \omega)=0,
$$

Using the exponential substitution $\xi=e^{-b z / 2}$ to equation (8). We obtain

$$
\xi^{2} \frac{d^{2} \widetilde{E}_{o v}}{d \xi^{2}}+\xi \frac{d \widetilde{E}_{o v}}{d \xi}-\frac{4}{b^{2}} \lambda^{2} \widetilde{E}_{o v}=-\frac{4}{b^{2}} k_{o v}^{2} \widetilde{E}_{o v}
$$

Equation (9) is a non-homogeneous differential equation, it can be solved for a homogeneous equation by Cauchy-Euler equation method and a particular solution by the method of variation of parameter. Hence, the solution of equation (9) is

$$
\begin{aligned}
& \widetilde{E}_{o v}(\lambda, \xi, \omega) \\
& =\kappa_{1} \xi^{\alpha}+\kappa_{2} \xi^{-\alpha}+\frac{i 2 \omega \mu_{g}}{\alpha b^{2}} \int_{0}^{1} G(\xi ; \tau) R(\tau) d \tau, 0 \leq z \leq d,
\end{aligned}
$$

where $R(\tau)=\int_{0}^{\infty} s\left[\sigma_{0}-(1 / b) 2 \ln \tau\right] E_{o v}(s, \tau, \omega) J_{1}(\lambda s) d s$, and $G(\xi ; \tau)$

$=\tau^{\alpha} \xi^{-\alpha}$ is the Green's function and $\alpha=2 \lambda / b$. We calculate the integration term of equation (10) by using method of subsection for which dividing the ground into $n$ layers and having the thickness equal to $d_{1}$, and assume the layer is very thin, so the current distribution on layer and Green's function for each layer may be approximated to be constant at each position of $z$. Therefore, equation (10) becomes

$$
\widetilde{E}_{o v}(\lambda, \xi, \omega)
$$




$$
=\kappa_{1} \xi^{\alpha}+\kappa_{2} \xi^{-\alpha}+\frac{i \omega \mu_{o v e}}{\alpha b} \sum_{j=1}^{n} G_{j}\left(\xi ; \xi_{j}\right) P\left(\lambda, \xi_{j}, \omega\right)\left(\xi_{j}\right), 0 \leq z \leq d
$$

where $\kappa_{1}, \kappa_{2}$ are arbitrary constants to be determined from the boundary condition, and $P\left(\lambda, \xi_{j}, \omega\right)=\int_{0}^{\infty} s p\left(s, \xi_{j}, \omega\right) J_{1}(\lambda s) d s$ such that $p\left(s, \xi_{j}, \omega\right)=d\left[\sigma_{0}-\right.$ $\left.(2 / b) \ln \xi_{j}\right] E_{\text {jove }}\left(s, \xi_{j}, \omega\right)$.

We can obtain partial differential equation for the electric filed in host medium from equation (4) as

$$
\begin{aligned}
& \frac{\partial^{2} E_{h o}(r, z, \omega)}{\partial z^{2}}+\frac{\partial^{2} E_{h o}(r, z, \omega)}{\partial r^{2}} \\
& +\frac{1}{r} \frac{\partial E_{h o}(r, z, \omega)}{\partial r}-\frac{E_{h o}(r, z, \omega)}{r^{2}}+k_{h o}^{2} E_{h o}(r, z, \omega)=0 .
\end{aligned}
$$

where $E_{h o}(r, z, \omega)$ is the electric field in host medium, $k_{h}^{2}=i \omega \mu_{h} \sigma_{0}+\omega^{2} \mu_{h} \varepsilon_{h}$, $\mu_{h}$ is the magnetic permeability of host medium, $\varepsilon_{h}$ is the electric permittivity of overburden and $\sigma_{0}$ is the conductivity of host medium. Taking Hankel transform to equation (12), we have

$$
\frac{d^{2} \widetilde{E}_{h o}(\lambda, z, \omega)}{d z^{2}}-m_{h}^{2} \widetilde{E}_{h o}(\lambda, z, \omega)=0
$$

where $m_{h}^{2}=\lambda^{2}-k_{h}^{2}$. The solution of equation (13) is

$$
\widetilde{E}_{h o}(\lambda, z, \omega)=\kappa_{3} e^{-m_{h} z}+\kappa_{4} e^{m_{h} z},
$$

where $\kappa_{3}, \kappa_{4}$ are arbitrary constants to be determined from the boundary conditions. Under the condition that $z \rightarrow \infty$, then $\widetilde{E}_{h o} \rightarrow 0$, we require that $\kappa_{4}=0$. Consequently, equation (14) becomes

$$
\widetilde{E}_{h o}(\lambda, z, \omega)=\kappa_{3} e^{-m_{h} z}, \quad z \geq d
$$

The constants $\kappa$ from equation (6), $\kappa_{1}, \kappa_{2}$ from equation (11), and $\kappa_{3}$ from equation (15) can be solved by imposing the continuity of $\widetilde{E}$ and $\frac{\partial \widetilde{E}}{\partial z}$ at the interface. Therefore, we obtain the electric fields in air as

$$
\begin{aligned}
& E_{a i}(r, z, \omega) \\
& =i \omega \mu_{0} a I(\omega) \int_{0}^{\infty}\left(\frac{e^{-\lambda|z+h|}}{\sqrt{8}}+\frac{e^{-\lambda|h|-2 \lambda d+\lambda z}}{\sqrt{8}\left[m_{h}+\lambda\right]}\right) J_{1}(\lambda a) J_{1}(\lambda r) d \lambda
\end{aligned}
$$




$$
+\frac{i \omega \mu_{\text {ove }}}{\sqrt{8}} \int_{0}^{\infty}\left[e^{\lambda d}-e^{\lambda z}\right] \sum_{j=1}^{n} P\left(\lambda, \xi_{j}, \omega\right)\left(\xi_{j}\right)^{\frac{2 \lambda}{b}} J_{1}(\lambda r) d \lambda .
$$

The electric fields in overburden as

$$
\begin{aligned}
& E_{\text {ov }}(r, z, \omega) \\
& =\frac{i \omega \mu_{0} a I(\omega)}{\sqrt{8}} \int_{0}^{\infty}\left(e^{-\lambda z}+\frac{e^{-2 \lambda d}}{\left[m_{h}+\lambda\right]} e^{\lambda z}\right) e^{-\lambda|h|} J_{1}(\lambda a) J_{1}(\lambda r) d \lambda \\
& +\frac{i \omega \mu_{\text {ove }}}{\sqrt{8}} \int_{0}^{\infty}\left[e^{\lambda d}-e^{\lambda z}\right] \sum_{j=1}^{n} P\left(\lambda, \xi_{j}, \omega\right)\left(\xi_{j}\right)^{\frac{2 \lambda}{b}} J_{1}(\lambda r) d \lambda .
\end{aligned}
$$

and the electric fields in host medium as

$$
\begin{aligned}
& E_{h o}(r, z, \omega) \\
& =\int_{0}^{\infty}\left(\frac{m_{h}+\lambda+1}{\sqrt{8}\left[m_{h}+\lambda\right]}\right) e^{m_{h} d-m_{h} z-\lambda d-\lambda|h|} J_{1}(\lambda a) J_{1}(\lambda r) d \lambda \\
& \quad+\frac{i \omega \mu_{\text {ove }}}{\sqrt{8}} \int_{0}^{\infty}\left[e^{\lambda d}-1\right] \sum_{j=1}^{n} P\left(\lambda, \xi_{j}, \omega\right)\left(\xi_{j}\right)^{\frac{2 \lambda}{b}} J_{1}(\lambda r) d \lambda .
\end{aligned}
$$

The electric field on the ground surface can be determined from equations (16) or equation (17) by taking $z=0$. Chave's algorithm [1] is used for numerical calculating the inverse Hankel transform of the electric field solutions.

\section{Conclusions}

In this paper, we present the method to study the structure of the earth's surface layers. The integral expressions are used to produce the forward modeling. We begin by formulating the problem to get the electric fields which could be used to find the electric fields on the ground surface. We consider two layered earth model having a positively skewed curve conductivity profile on the first layer which is denoted by $\sigma_{o v}(z)=\left(\sigma_{0}+z\right) e^{-b z / 2}, 0 \leq z \leq d$, where $\sigma_{0}$ is a positive constant, $b$ is constant, $d$ is the thickness of overburden. The conductivity of host medium, $z \geq d$, is a constant given by $\sigma_{h o}(z)=\sigma_{0}$ on the second layer. The graphs are shown the behavior of the electric field against source-receiver 


\begin{tabular}{|c|c|c|}
\hline$r$ & $R E A L b=0.5 d=1$ & IM $b=0.5 d=1$ \\
\hline 0.1 & $-3.15 E-03$ & $3.95 E-03$ \\
\hline 0.2 & $-6.25 E-03$ & $7.87 E-03$ \\
\hline 0.3 & $-9.23 E-03$ & $1.17 E-02$ \\
\hline 0.4 & $-1.21 E-02$ & $1.55 E-02$ \\
\hline 0.5 & $-1.47 E-02$ & $1.91 E-02$ \\
\hline 0.6 & $-1.70 E-02$ & $2.26 E-02$ \\
\hline 0.7 & $-1.91 E-02$ & $2.60 E-02$ \\
\hline 0.8 & $-2.09 E-02$ & $2.91 E-02$ \\
\hline 0.9 & $-2.23 E-02$ & $3.21 E-02$ \\
\hline 1 & $-2.34 E-02$ & $3.48 E-02$ \\
\hline 1.1 & $-2.41 E-02$ & $3.73 E-02$ \\
\hline 1.2 & $-2.45 E-02$ & $3.96 E-02$ \\
\hline 1.3 & $-2.45 E-02$ & $4.16 E-02$ \\
\hline 1.4 & $-2.43 E-02$ & $4.34 E-02$ \\
\hline 1.5 & $-2.37 E-02$ & $4.50 E-02$ \\
\hline 1.6 & $-2.29 E-02$ & $4.64 E-02$ \\
\hline 1.7 & $-2.19 E-02$ & $4.75 E-02$ \\
\hline 1.8 & $-2.06 E-02$ & $4.84 E-02$ \\
\hline 1.9 & $-1.92 E-02$ & $4.91 E-02$ \\
\hline
\end{tabular}

Figure 2: Chave's algorithm [1] is used for numerical calculating the inverse hankel transform by setting $\sigma_{0}=0.1 \mathrm{Sm}^{-1}, b=0.5 \mathrm{~m}^{-1}, d=$ $1 \mathrm{~m}$, frequency $=200 \mathrm{~Hz}$.
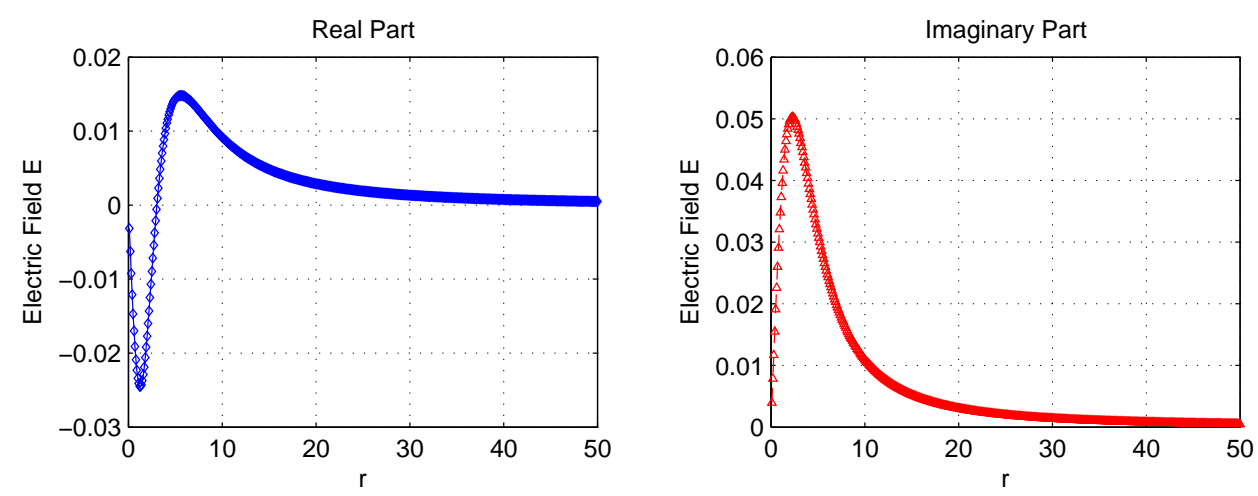

Figure 3: Graph of real and imaginary part of electric field $E$ versus $r$ for a two layered ground model with a positively skewed curve medium by inputting $\sigma_{0}=0.1 S \mathrm{~m}^{-1}, b=0.5 \mathrm{~m}^{-1}, d=1 \mathrm{~m}$, frequency $=200$ $\mathrm{Hz}$. 

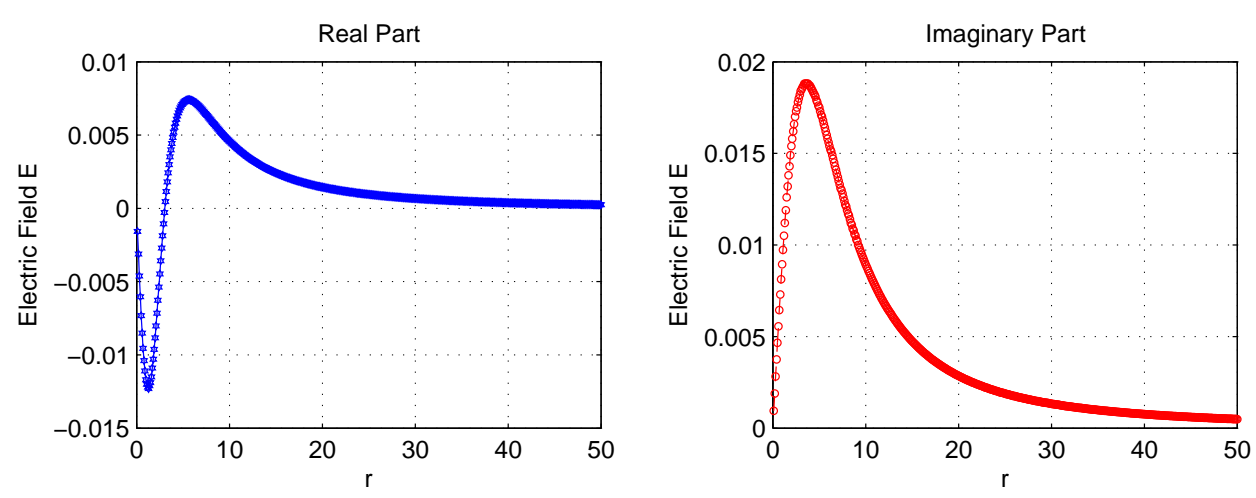

Figure 4: Graph of real and imaginary part of electric field $E$ versus $r$ for a two layered ground model with a positively skewed curve medium by inputting $\sigma_{0}=0.1 \mathrm{~S} \mathrm{~m}^{-1}, b=0.5 \mathrm{~m}^{-1}, d=5.5 \mathrm{~m}$, frequency $=100$ $\mathrm{Hz}$.

spacing $r$ at different depths. The values of $d$ and frequency are varied while the values of $\sigma_{0}$ and $b$ are fixed. The curves of the electric fields are similar to the figure of a positively skewed curve conductivity ground profile as shown in figures. The graphs that we obtained are oscillated first and tend to zero which is resulted by the conductivity profile. The inversion process will be studied in the near future work to find out the optimal values for all the parameters.

\section{References}

[1] A.D. Chave, " Numerical integration of related Hankel transforms by quadrature and continued fraction expansion", Geophysics 48(1983), 16711686.

[2] G. W. Hohmann and A. P. Raiche, Inversion of controlled-Source Electromagnetic Data, Electromagnetic methods in applied geophysics Vol. 1(1988), 469-503.(ed. Nabighian, M. N.)SEG

[3] T.J. Lee and R. Ignetik, Transient Electromagnetic Response of a halfspace with Exponential Conductivity Profile and its Applications to Salinity Mapping, Exploration Geophysics 25(1994), 39-51. 
[4] H.F. Morrison, R.J. Phillips and D.P. O'Brien, Quantitative interpretation of transient electromagnetic fields over a layered half-space, Geophysical prospecting, 21(1969), 1-20.

[5] S. Yooyuanyong, Inversion by EM sounding for a disk embedded in a conducting half-space, Songklanakarin J. Sci. Technol. 21(2)(1999), 197-205.

[6] P. Haarsa, Electromagnetic response on a half-space earth with a positively skewed bulge conductivity, Int. J. Applied Math. Stat., 42(12)(2013), 324329.

[7] P. Haarsa and S. Pothat, Mathematical model of electromagnetic response from a positively skewed bulge ground structure, Applied Math. Sci., 7(101)(2013), 5013-5023.

[8] P. Haarsa and S. Pothat, On Taylor-Series Approximation of the Response of Electromagnetic Field on a Two Layered Earth Model with a Conductive Overburden, Int. J. Pure Appl. Math., 89(5)(2013), 699-709. 
